\title{
Origin of the exponential decay of the Loschmidt echo in integrable systems
}

\author{
Rémy Dubertrand ${ }^{1,2}$ and Arseni Goussev ${ }^{3,4}$ \\ ${ }^{1}$ Universite de Toulouse; UPS, Laboratoire de Physique Theorique (IRSAMC); F-31062 Toulouse, France \\ ${ }^{2}$ CNRS, LPT (IRSAMC), F-31062 Toulouse, France \\ ${ }^{3}$ Department of Mathematics and Information Sciences, Northumbria University, Newcastle Upon Tyne, NE1 8ST, United Kingdom \\ ${ }^{4}$ Max Planck Institute for the Physics of Complex Systems, Nöthnitzer Straße 38, D-01187 Dresden, Germany
}

(Received 11 December 2013; published 18 February 2014)

\begin{abstract}
We address the time decay of the Loschmidt echo, measuring the sensitivity of quantum dynamics to small Hamiltonian perturbations, in one-dimensional integrable systems. Using a semiclassical analysis, we show that the Loschmidt echo may exhibit a well-pronounced regime of exponential decay, similar to the one typically observed in quantum systems whose dynamics is chaotic in the classical limit. We derive an explicit formula for the exponential decay rate in terms of the spectral properties of the unperturbed and perturbed Hamilton operators and the initial state. In particular, we show that the decay rate, unlike in the case of the chaotic dynamics, is directly proportional to the strength of the Hamiltonian perturbation. Finally, we compare our analytical predictions against the results of a numerical computation of the Loschmidt echo for a quantum particle moving inside a one-dimensional box with Dirichlet-Robin boundary conditions, and find the two in good agreement.
\end{abstract}

DOI: 10.1103/PhysRevE.89.022915

PACS number(s): 05.45.Mt, 03.65.Sq, 03.65.Yz

\section{INTRODUCTION}

Even a tiny perturbation of the Hamiltonian of a quantum systems may significantly alter its time evolution. Changes in the dynamics of the corresponding unperturbed and perturbed systems can be conveniently quantified in terms of the Loschmidt echo (LE) that is defined as

$$
M(t)=|m(t)|^{2},
$$

with

$$
m(t)=\left\langle\Psi_{\lambda_{2}}(t) \mid \Psi_{\lambda_{1}}(t)\right\rangle
$$

being the overlap between two quantum states, $\left|\Psi_{\lambda_{1}}(t)\right\rangle$ and $\left|\Psi_{\lambda_{2}}(t)\right\rangle$, resulting from the same initial state $\left|\Psi_{\lambda_{1}}(0)\right\rangle=$ $\left|\Psi_{\lambda_{2}}(0)\right\rangle=\left|\Psi_{0}\right\rangle$ in the course of the time evolution under different Hamilton operators, $H_{\lambda_{1}}$ and $H_{\lambda_{2}}$, respectively. The LE equals 1 at $t=0$ and is typically smaller than 1 at $t>0$. Over the past two decades, the time decay of the LE has been addressed, both experimentally and theoretically, in a large variety of quantum systems with nontrivial, complex dynamics, and has been proven to be an invaluable tool in understanding dynamical properties of these systems. A vast body of literature on the subject is summarized in review articles [1-3].

Most of the attention on the LE has been directed at quantum systems whose dynamics is chaotic in the classical limit. This has to do with the existence of a parametric regime, commonly referred to as the Lyapunov regime, in which the LE of a chaotic quantum system decays exponentially in time, with the decay rate given by the average Lyapunov exponent of the underlying classical system [4-7]. The Lyapunov regime makes the LE a valuable tool for identifying signatures of chaotic behavior in the dynamics of quantum systems.

In integrable (regular) systems, unlike in chaotic systems, the decay of the LE remains far less understood. Two robust decay regimes have been established analytically and observed in numerical simulations. The first regime is characterized by a Gaussian decay of the LE, $M(t) \sim \exp \left(-\right.$ const $\left.\times t^{2}\right)$.
It occurs under sufficiently weak Hamiltonian perturbations and takes place on a time scale inversely proportional to the perturbation strength [8]. The second regime exhibits an algebraic decay of the LE, $M(t) \sim t^{-3 d / 2}$, with $d$ being the dimensionality of the system. The algebraic decay occurs when the Hamiltonian perturbation is sufficiently strong and varies rapidly along a typical classical trajectory of the system [9]. In general, however, the time decay of the LE in integrable systems is nonmonotonic, may be accompanied by revivals [10] and intervals of temporary freeze [11], and may exhibit sharp minima and maxima on short-time scales [12].

Surprisingly, numerical simulations have shown that, in addition to the Gaussian and algebraic decays, integrable systems may also exhibit exponential decay of the LE $[13,14]$. This finding may seem unexpected as the exponential decay of the LE is normally regarded as a hallmark of chaotic dynamics. To our knowledge, a theoretical model that is able to quantitatively explain exponential decay of the LE in integrable systems is lacking.

In this paper, we show analytically that exponential decay of the LE may occur even in the simplest, one-dimensional integrable systems. For such systems, we derive an explicit formula giving the exponential decay rate in terms of spectral characteristics of the unperturbed and perturbed Hamilton operators and the initial state. In particular, we show that the decay rate is directly proportional to the strength of the Hamiltonian perturbation. This linear dependence is to be compared with the corresponding rate-versus-strength dependence in the case of chaotic dynamics: Under weak perturbations, the decay rate is quadratic in the perturbation strength (the Fermi-golden-rule regime) [5], while under strong perturbations, the decay rate is independent of the perturbation strength (the Lyapunov regime) [4]. Finally, using an example system-a quantum particle moving inside a one-dimensional box with Dirichlet-Robin boundary conditions-we compare our analytical prediction against the exact (numerically computed) LE, and find the two in good agreement. 


\section{THEORY OF THE EXPONENTIAL DECAY IN ONE-DIMENSIONAL SYSTEMS}

Let $H_{\lambda}$ be a family of integrable one-dimensional Hamiltonians, parametrized by some parameter $\lambda$. The eigenvalues and eigenvectors of $H_{\lambda}$ are respectively denoted by $E_{\lambda}(n)$ and $\left|\psi_{\lambda}(n)\right\rangle$, where $n$ stands for the quantum number of the one-dimensional system.

In what follows, we consider a parametric regime of asymptotically small perturbations, in which $\lambda_{1}=\lambda$ and $\lambda_{2}=\lambda+\epsilon$ with $|\epsilon| \ll|\lambda|$. The LE amplitude $m(t)$, defined by Eq. (2), can be expressed as a double sum over the eigenstates of both $H_{\lambda}$ and $H_{\lambda+\epsilon}$. Using the diagonal approximation, corresponding to the leading-order perturbation theory, one arrives at the standard, single sum approximation to the LE amplitude [15]:

$$
m(t) \simeq \sum_{n}\left|\left\langle\psi_{\lambda}(n) \mid \Psi_{0}\right\rangle\right|^{2} e^{i\left[E_{\lambda+\epsilon}(n)-E_{\lambda}(n)\right] t / \hbar} .
$$

Infinite sums, mathematically similar to the one in Eq. (3), are commonly encountered in studies of time-domain autocorrelation functions of quantum wave packets $[16,17]$.

We analyze the sum in Eq. (3) in the semiclassical limit of high energies. Motivated by the study of quantum revivals of the autocorrelation function in Ref. [16], we consider an initial state that is a linear superposition of a large number of highly excited states:

$$
\left|\left\langle\psi_{\lambda}(n) \mid \Psi_{0}\right\rangle\right|^{2} \simeq \frac{1}{\sqrt{2 \pi(\Delta n)^{2}}} \exp \left[\frac{-\left(n-n_{0}\right)^{2}}{2(\Delta n)^{2}}\right],
$$

with

$$
1 \ll \Delta n \ll n_{0} .
$$

We note that, in view of the condition (5), the initial state is normalized to unity, $\sum_{n}\left|\left\langle\psi_{\lambda}(n) \mid \Psi_{0}\right\rangle\right|^{2} \simeq 1$.

We now assume that, for large enough values of the quantum number, the energy levels can be asymptotically expanded as

$$
E_{\lambda}(n) \simeq h(n)+g(\lambda)+f(\lambda) n^{\nu}, \quad n \gg 1 .
$$

Here, the terms $h(n)$ and $g(\lambda)$ are, respectively, functions of $n$ and $\lambda$ only. The term $f(\lambda) n^{\nu}$ depends on both $n$ and $\lambda$, and is the first, leading-order term of the expansion (in powers of $1 / n$ ) whose coefficient depends on $\lambda$. The exponent $v$ is a nonzero real number. We note that it is the term $f(\lambda) n^{v}$ that will play a crucial role in the following analysis.

Expansion (6) approximates the spectrum of a rather broad class of quantum systems, whose energy levels in the semiclassical (high-energy) regime can be expanded into a power series in the quantum number. Some examples are the motion of a quantum particle confined to the potential well $V(x)=V_{0}|x|^{\alpha}$ with any real positive $\alpha$, including the harmonic oscillator, and the radial motion of an electron in the hydrogenlike atom. Furthermore, we only consider smooth perturbations that do not change the power series structure of the energy spectrum.

In view of Eq. (6), the phase difference in Eq. (3) becomes

$$
E_{\lambda+\epsilon}(n)-E_{\lambda}(n) \simeq \epsilon g^{\prime}(\lambda)+\epsilon f^{\prime}(\lambda) n^{\nu},
$$

where the prime denotes the derivative. The time scale for which this approximation holds coincides with the range of validity of the perturbative regime. Then, expanding the righthand side of Eq. (7) around $n_{0}$, we obtain

$$
\begin{aligned}
& E_{\lambda+\epsilon}(n)-E_{\lambda}(n) \\
& \quad \simeq \epsilon g^{\prime}(\lambda)+\epsilon f^{\prime}(\lambda) n_{0}^{v} \sum_{k=0}^{\infty} \frac{\Gamma(v+1)}{\Gamma(v-k+1) k !}\left(\frac{n-n_{0}}{n_{0}}\right)^{k},
\end{aligned}
$$

where $\Gamma$ denotes the Euler Gamma function generalizing the factorial.

It is instructive to note that some well-known results for the LE decay can be recovered by truncating the infinite sum in Eq. (8) and substituting the truncated sum into Eq. (3). The Gaussian decay regime is obtained if one only keeps terms up to the linear one in $\left(n-n_{0}\right) / n_{0}$, i.e., the two terms corresponding to $k=0$ and $k=1$. Retaining additionally the quadratic term $k=2$ leads to an algebraic modification of the variance in the Gaussian regime. As we will show below, the cubic term $k=3$ gives rise to a new asymptotic decay regime, which follows the (algebraically modified) Gaussian decay, and in which the LE decays essentially exponentially in time.

Substituting Eqs. (4) and (8), truncated at $k=3$, into Eq. (3), and replacing the sum by the corresponding integral, we obtain

$$
m(t) \simeq e^{i \phi} \int_{-\infty}^{\infty} \exp \left(i a n^{3}-b n^{2}+i c n\right) \frac{d n}{\sqrt{2 \pi(\Delta n)^{2}}},
$$

with $\phi=\epsilon g^{\prime}(\lambda) t / \hbar$,

$$
\begin{aligned}
a & =\frac{\nu(\nu-1)(\nu-2)}{6} n_{0}^{\nu-3} \tau, \\
b & =\frac{1}{2(\Delta n)^{2}}-\frac{i}{2} \nu(\nu-1) n_{0}^{\nu-2} \tau, \\
c & =v n_{0}^{\nu-1} \tau,
\end{aligned}
$$

and

$$
\tau=\frac{\epsilon f^{\prime}(\lambda) t}{\hbar} .
$$

The integral in Eq. (9) can be evaluated using the identity [18]

$$
\begin{aligned}
\int_{-\infty}^{\infty} e^{i a x^{3}-b x^{2}+i c x} d x= & \frac{2 \pi}{|3 a|^{\frac{1}{3}}} \exp \left(\frac{b c}{3 a}+\frac{2 b^{3}}{27 a^{2}}\right) \\
& \times \operatorname{Ai}\left[\frac{\operatorname{sgn}(a)}{|3 a|^{\frac{1}{3}}}\left(\frac{b^{2}}{3 a}+c\right)\right],
\end{aligned}
$$

valid for real $a$ and $c$, and $\operatorname{Re} b>0$. Here, Ai denotes the Airy function and $\operatorname{sgn}(x)$ is the sign function, defined as $\operatorname{sgn}(x)=1$ for $x>0$ and $\operatorname{sgn}(x)=-1$ for $x<0$. Focusing on the time scale beyond the validity range of the Gaussian decay regime,

$$
\tau \gg n_{0}^{2-v}(\Delta n)^{-2},
$$


and using the identity (12) in Eq. (9), we obtain, to the leading order in $n_{0}^{2-v}(\Delta n)^{-2} / \tau$,

$$
\begin{aligned}
|m(t)| \simeq & \sqrt{\frac{2 \pi}{(\Delta n)^{2}}\left|\frac{2}{v(v-1)(v-2) n_{0}^{v-3} \tau}\right|^{\frac{1}{3}}} \\
& \times \exp \left[-\frac{1}{(v-1)(v-2)^{2}}\left(\frac{n_{0}}{\Delta n}\right)^{2}\right] \\
& \times \operatorname{Ai}\left[\operatorname{sgn}\left(\frac{v-3}{v-1}\right) \frac{|v-3|}{|v-1|^{\frac{1}{3}}}\left(\frac{|v| n_{0}^{v} \tau}{2(v-2)^{2}}\right)^{\frac{2}{3}}\right] .
\end{aligned}
$$

We now assume that $v<1$, which guarantees the argument of the Airy function in Eq. (14), be strictly positive. Then, in view of conditions (5) and (13), we use the large argument asymptotics of the Airy function [19],

$$
\operatorname{Ai}(x) \simeq \frac{1}{2 \sqrt{\pi} x^{\frac{1}{4}}} \exp \left(-\frac{2}{3} x^{\frac{3}{2}}\right), \quad x \gg 1,
$$

and obtain the following approximate expression for the LE:

$$
M(t) \simeq \frac{A}{t} e^{-\gamma t},
$$

with

$$
\gamma=\frac{2|\nu|}{3(v-2)^{2}} \sqrt{\frac{|v-3|^{3}}{|v-1|}} \frac{\left|\epsilon f^{\prime}(\lambda)\right| n_{0}^{v}}{\hbar}
$$

and

$$
\begin{aligned}
A= & \frac{n_{0}^{2-v}(\Delta n)^{-2} \hbar}{\sqrt{v^{2}(v-1)(v-3)}\left|\epsilon f^{\prime}(\lambda)\right|} \\
& \times \exp \left[-\frac{2}{(v-1)(v-2)^{2}}\left(\frac{n_{0}}{\Delta n}\right)^{2}\right] .
\end{aligned}
$$

Equations (16)-(18) constitute the main analytical result of the present paper. Equation (16) shows that the LE may decay exponentially in time (not taking into account an algebraic prefactor) even in quantum systems whose counterpart classical dynamics is not chaotic, and as simple as that of a one-dimensional conservative system.

\section{PARTICLE IN A BOX WITH DIRICHLET-ROBIN BOUNDARY CONDITIONS}

We now illustrate our theory by considering the dynamics of a quantum particle trapped inside a one-dimensional box, $0<x<1$. The particle state $|\Psi(t)\rangle$ evolves according to the free-particle Schrödinger equation,

$$
\left(i \partial_{t}+\partial_{x}^{2}\right)\left\langle x \mid \Psi_{\lambda}(t)\right\rangle=0,
$$

subject to a Dirichlet boundary condition at $x=0$ and a Robin boundary condition at $x=1$ (see Fig. 1),

$$
\begin{gathered}
\left.\left\langle x \mid \Psi_{\lambda}(t)\right\rangle\right|_{x=0}=0, \\
\left.\left(\partial_{x}+\lambda\right)\left\langle x \mid \Psi_{\lambda}(t)\right\rangle\right|_{x=1}=0 .
\end{gathered}
$$

Hereinafter, we set $\hbar=1$ and $m=1 / 2$. We address the time decay of the LE, defined by Eq. (1), due to a small perturbation

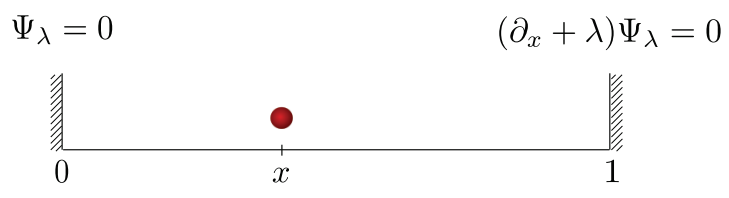

FIG. 1. (Color online) Particle in a one-dimensional box with Dirichlet-Robin boundary conditions.

of the real-valued control parameter $\lambda$, i.e., $\lambda_{1}=\lambda$ and $\lambda_{2}=\lambda+\epsilon$ specify the unperturbed and perturbed systems, respectively. We take the initial state $\left|\Psi_{\lambda_{1}}(0)\right\rangle=\left|\Psi_{\lambda_{2}}(0)\right\rangle=$ $\left|\Psi_{0}\right\rangle$ to be given by a Gaussian wave packet

$$
\left\langle x \mid \Psi_{0}\right\rangle=\left(\frac{1}{\pi \sigma^{2}}\right)^{\frac{1}{4}} \exp \left(i p_{0}\left(x-x_{0}\right)-\frac{\left(x-x_{0}\right)^{2}}{2 \sigma^{2}}\right) .
$$

Here, $x_{0}$ and $p_{0}$ correspond to the initial average position and momentum of the particle, respectively, and $\sigma$ quantifies the spatial dispersion of the wave packet. Provided $0<x_{0}<1$ and $\sigma \ll x_{0}\left(1-x_{0}\right)$, the entire probability density is well localized within the box and the boundary conditions (20) and (21) are satisfied with exponentially high accuracy. We use the values $x_{0}=0.5$ and $\sigma=0.01$ in all our numerical examples.

Eigenstates $\left|\psi_{\lambda}(n)\right\rangle$ and energy levels $E_{\lambda}(n) \equiv z_{\lambda}^{2}(n)$ of the system are determined by the equation

$$
\left[\partial_{x}^{2}+z_{\lambda}^{2}(n)\right]\left\langle x \mid \psi_{\lambda}(n)\right\rangle=0 \text { for } n \in \mathbb{N},
$$

along with the boundary conditions (20) and (21). Written out explicitly, the orthonormal eigenstates are

$$
\left\langle x \mid \psi_{\lambda}(n)\right\rangle=\sqrt{2}\left[1-\frac{\sin \left[2 z_{\lambda}(n)\right]}{2 z_{\lambda}(n)}\right]^{-\frac{1}{2}} \sin \left[z_{\lambda}(n) x\right],
$$

where the energy levels are determined by solutions of the transcendental equation

$$
z_{\lambda}(n) \cos z_{\lambda}(n)+\lambda \sin z_{\lambda}(n)=0 .
$$

Equation (25) can be straightforwardly solved numerically, to a high degree of accuracy, for any desired range of $n$. For our purposes, it proved sufficient to compute the first 350 eigenstates and eigenlevels of the unperturbed and perturbed systems.

In terms of the eigenstates and eigenlevels, the expression for the LE takes the form

$$
\begin{aligned}
M(t)= & \mid \sum_{n, m}\left\langle\Psi_{0} \mid \psi_{\lambda_{2}}(m)\right\rangle\left\langle\psi_{\lambda_{2}}(m) \mid \psi_{\lambda_{1}}(n)\right\rangle \\
& \times\left.\left\langle\psi_{\lambda_{1}}(n) \mid \Psi_{0}\right\rangle e^{-i\left[z_{\lambda_{1}}^{2}(n)-z_{\lambda_{2}}^{2}(m)\right] t}\right|^{2} .
\end{aligned}
$$

Here, the amplitude of the overlap between the unperturbed and perturbed eigenstates can be written, using Eq. (24) together with the boundary conditions (20) and (21), as

$$
\left\langle\psi_{\lambda_{2}}(m) \mid \psi_{\lambda_{1}}(n)\right\rangle=\left(\lambda_{1}-\lambda_{2}\right) \frac{\left\langle 1 \mid \psi_{\lambda_{1}}(n)\right\rangle\left\langle\psi_{\lambda_{2}}(m) \mid 1\right\rangle}{z_{\lambda_{1}}^{2}(n)-z_{\lambda_{2}}^{2}(m)} .
$$

We note that, for a sufficiently weak perturbation $\epsilon$, the right-hand side of Eq. (27) can be well approximated by the 


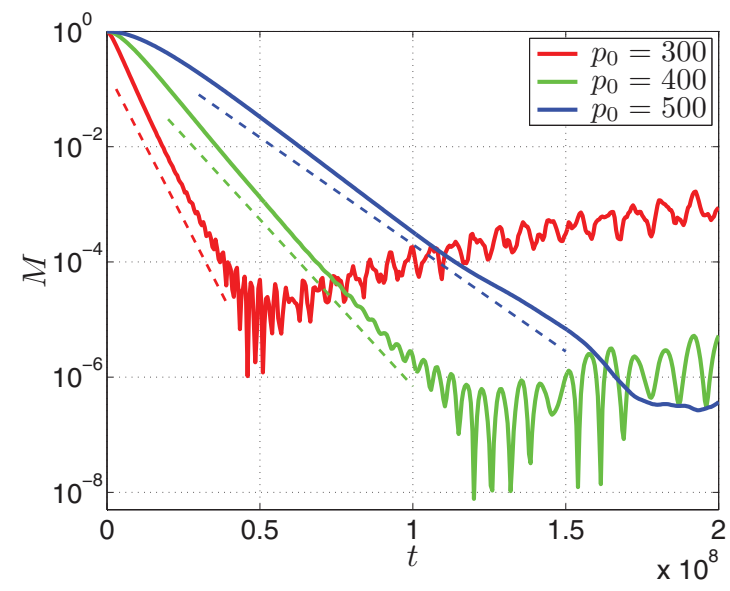

FIG. 2. (Color online) Time decay of the LE for a particle moving inside a one-dimensional box with Dirichlet-Robin boundary conditions. The system, perturbation, and the initial state are characterized by the following set of parameters: $\lambda=1, \epsilon=0.01, x_{0}=0.5$, $\sigma=0.01$, and $p_{0}=300$ (red), 400 (green), 500 (blue). Solid curves represent the numerically exact LE. Straight dashed lines of the corresponding color show the trend of the exponential decay $e^{-\gamma t}$, with $\gamma$ computed in accordance with Eqs. (37). See text for details.

Kronecker's symbol $\delta_{m, n}$, and, consequently, the expression for the LE amplitude can be reduced to Eq. (3). More accurately, however, the value of the LE, $M(t)$, can be obtained by substituting Eqs. (22), (24), and (27) into Eq. (26), and numerically evaluating the overlap amplitudes $\left\langle\psi_{\lambda}(n) \mid \Psi_{0}\right\rangle$ and the sums in Eq. (26).

Figure 2 presents three LE decay curves in the system with $\lambda=1$ under the perturbation $\epsilon=0.01$. The initial Gaussian state is specified by the average position $x_{0}=0.5$ and spatial dispersion $\sigma=0.01$. Three values of the average momentum are considered: $p_{0}=300$ (red solid curve), $p_{0}=400$ (green solid curve), and $p_{0}=500$ (blue solid curve). All three curves exhibit well-pronounced regions of nearly exponential decay featuring a LE drop over three to four orders of magnitude. It is interesting to note that the exponential decay persists over very-long-time intervals, corresponding to approximately $10^{9}-10^{10}$ periods of the underlying classical oscillation.

In order to compare the numerically obtained LE decay curves with the analytical predictions of the previous section, we evaluate the exponential decay rate $\gamma$ in accordance with Eq. (17). To this end, we expand the energy eigenlevels $E_{\lambda}(n)=z_{\lambda}^{2}(n)$ into a series of the form (6) for large $n$. We first write $z_{\lambda}$ in terms of a new quantity $\zeta_{\lambda}$ as

$$
z_{\lambda}(n)=\pi\left(n-\frac{1}{2}\right)+\zeta_{\lambda}(n) .
$$

Then, substituting Eq. (28) into Eq. (25), we obtain

$$
\left(\pi-n^{-1} \frac{\pi}{2}+n^{-1} \zeta_{\lambda}\right) \sin \zeta_{\lambda}=n^{-1} \lambda \cos \zeta_{\lambda}
$$

Since $\zeta_{\lambda} \rightarrow 0$ as $n^{-1} \rightarrow 0$, we look for $\zeta_{\lambda}$ in the form of the following power series in $n^{-1}$ :

$$
\zeta_{\lambda}=n^{-1} A_{\lambda}+n^{-2} B_{\lambda}+n^{-3} C_{\lambda}+O\left(n^{-4}\right)
$$

Substituting Eq. (30) into Eq. (29), expanding both sides of the resulting equation into power series in $n^{-1}$, and then matching the expansion coefficients on both sides of the equation, we find

$$
\begin{gathered}
A_{\lambda}=\frac{\lambda}{\pi}, \\
B_{\lambda}=\frac{\lambda}{2 \pi}, \\
C_{\lambda}=\frac{\lambda}{4 \pi}-\frac{\lambda^{2}}{\pi^{3}}-\frac{\lambda^{3}}{3 \pi^{3}} .
\end{gathered}
$$

Then, using Eqs. (30)-(33) into Eq. (28), we get

$$
\begin{aligned}
z_{\lambda}(n)= & \pi n-\frac{\pi}{2}+\frac{\lambda}{\pi n}+\frac{\lambda}{2 \pi n^{2}} \\
& +\left(\frac{\lambda}{4 \pi}-\frac{\lambda^{2}}{\pi^{3}}-\frac{\lambda^{3}}{3 \pi^{3}}\right) n^{-3}+O\left(n^{-4}\right) .
\end{aligned}
$$

Raising $z_{\lambda}$ to the second power, we obtain

$$
E_{\lambda}(n)=\pi^{2}\left(n-\frac{1}{2}\right)^{2}+2 \lambda-\left(1+\frac{2}{3} \lambda\right) \frac{\lambda^{2}}{\pi^{2} n^{2}}+O\left(n^{-3}\right) .
$$

Comparing Eq. (35) with Eq. (6), we conclude that, for the system of a particle moving inside a one-dimensional Dirichlet-Robin box,

$$
v=-2 \text { and } f(\lambda)=-\left(1+\frac{2}{3} \lambda\right) \frac{\lambda^{2}}{\pi^{2}} .
$$

Finally, a substitution of Eq. (36) into Eq. (17) leads to the following explicit formula for the decay rate:

$$
\gamma=\frac{5}{6 \pi^{2}} \sqrt{\frac{5}{3}} \frac{|(1+\lambda) \lambda \epsilon|}{n_{0}^{2}} .
$$

The value of $n_{0}$ can be computed numerically as $n_{0}=$ $\sum_{n} n\left|\left\langle\psi_{\lambda}(n) \mid \Psi_{0}\right\rangle\right|^{2}$. For the three cases presented in Fig. 2, we obtain $n_{0} \simeq 96$ for the initial state with $p_{0}=300$ (red curve), $n_{0} \simeq 128$ for $p_{0}=400$ (green curve), and $n_{0} \simeq 160$ for $p_{0}=500$ (blue curve). A substitution of these values of $n_{0}$, along with $\lambda=1$ and $\epsilon=0.01$, yields the corresponding values of the decay rate $\gamma$. Dashed lines in Fig. 2 present the trend of the exponential decay $e^{-\gamma t}$ for each of the three cases. It is evident that Eq. (37) [or, equivalently, Eq. (17)] provides a very good estimate for the rate of the exponential decay of the LE.

Finally, we note that the quantum number dispersion $\Delta n$, evaluated as $(\Delta n)^{2}=\sum_{n}\left(n-n_{0}\right)^{2}\left|\left\langle\psi_{\lambda}(n) \mid \Psi_{0}\right\rangle\right|^{2}$, approximately equals 23 for all three initial states considered in this section. This value is consistent with condition (5), which is one of the central assumptions of our theory.

\section{DISCUSSION AND CONCLUSION}

In this paper we have developed an analytical theory of the long-time exponential decay of the Loschmidt echo (or fidelity) in one-dimensional integrable quantum systems under the action of integrable perturbations. In particular, we have shown that, in the integrable case, the rate of the exponential decay $\gamma$ is proportional to the perturbation strength $|\epsilon|$. This 
is to be contrasted with the $\gamma$ vs $\epsilon$ dependence typically observed in quantum systems whose dynamics is chaotic in the classical limit: $\gamma \propto|\epsilon|^{2}$ for weak perturbation (Fermi-goldenrule regime) and $\gamma \propto|\epsilon|^{0}$ for strong perturbations (Lyapunov regime). It is interesting to note that the linear dependence of the decay rate on the perturbation strength, $\gamma \propto|\epsilon|^{1}$, has been also observed in a quantum system with a chaotic classical counterpart under the action of a local perturbation [20]. We think it is important to explore the connection between this work and our results.

As a test of our analytical formulas we have applied our theory to the system of a quantum particle moving inside a one-dimensional box with Dirichlet boundary condition on one end and Robin boundary condition on the other. The Robin control parameter was used to induce a perturbation. We have shown the system to exhibit a well-pronounced regime of an exponential decay of the Loschmidt echo, and found a good agreement between the analytically predicted and numerically observed values of the decay rate.

Exponential decay of the Loschmidt echo in integrable systems has been previously addressed by numerical simulations. The exponential decay regime was clearly observed in Ref. [13], however, no clear relation between the decay rate and the perturbation strength was found. We attribute this to their particular choice of the initial state, at variance with the semiclassical condition (5). In Ref. [14], the exponential decay regime was found to be a transient from the shorttime Gaussian to the long-time power law decay. This is not in contradiction with our result. Our theory, in addition to explaining the physical origin of the exponential decay regime, provides an explicit formula for the decay rate, and, in particular, gives the dependence of the decay rate on the perturbation strength.

More generally, the current study emphasizes the benefit of using the Loschmidt echo as a tool for analyzing the longtime asymptotics of quantum dynamics in the semiclassical regime. We have found that the Loschmidt echo may decay exponentially even in one-dimensional quantum systems. This can be related to the structural instability of the corresponding classical integrable systems. A natural way to extend our approach is to address higher-dimensional systems. One of the central challenges of such a study is the necessity to account for quasidegeneracies of the energy spectrum, generic in many-dimensional integrable systems.

\section{ACKNOWLEDGMENTS}

R.D. acknowledges financial support by Programme Investissements d'Avenir under the program ANR-11-IDEX0002-02, reference No. ANR-10-LABX-0037-NEXT (ENCOQUAM project). A.G. thanks EPSRC for support under Grant No. EP/K024116/1.
[1] T. Gorin, T. Prosen, T. H. Seligman, and M. Znidaric, Phys. Rep. 435, 33 (2006).

[2] Ph. Jacquod and C. Petitjean, Adv. Phys. 58, 67 (2009).

[3] A. Goussev, R. A. Jalabert, H. M. Pastawski, and D. A. Wisniacki, Scholarpedia 7, 11687 (2012).

[4] R. A. Jalabert and H. M. Pastawski, Phys. Rev. Lett. 86, 2490 (2001).

[5] Ph. Jacquod, P. G. Silvestrov, and C. W. J. Beenakker, Phys. Rev. E 64, 055203(R) (2001).

[6] F. M. Cucchietti, H. M. Pastawski, and D. A. Wisniacki, Phys. Rev. E 65, 045206(R) (2002).

[7] F. M. Cucchietti, H. M. Pastawski, and R. A. Jalabert, Phys. Rev. B 70, 035311 (2004).

[8] T. Prosen, Phys. Rev. E 65, 036208 (2002).

[9] Ph. Jacquod, I. Adagideli, and C. W. J. Beenakker, Europhys. Lett. 61, 729 (2003)

[10] R. Sankaranarayanan and A. Lakshminarayan, Phys. Rev. E 68, 036216 (2003).
[11] T. Prosen and M. Žnidarič, New J. Phys. 5, 109 (2003).

[12] A. Goussev, Phys. Rev. E 83, 056210 (2011).

[13] Y. S. Weinstein and C. S. Hellberg, Phys. Rev. E 71, 016209 (2005).

[14] W.-ge Wang, G. Casati, and B. Li, Phys. Rev. E 75, 016201 (2007).

[15] A. Peres, Phys. Rev. A 30, 1610 (1984).

[16] M. Nauenberg, J. Phys. B: At. Mol. Opt. Phys. 23, L385 (1990).

[17] R. W. Robinett, Phys. Rep. 392, 1 (2004).

[18] See, e.g., C. Leichtle, I. Sh. Averbukh, and W. P. Schleich, Phys. Rev. A 54, 5299 (1996).

[19] I. S. Gradshteyn and I. M. Ryzhik, Table of Integrals, Series, and Products, edited by A. Jeffrey and D. Zwillinger (Academic, New York, 2007), 7th ed.

[20] D. A. Wisniacki, E. G. Vergini, H. M. Pastawski, and F. M. Cucchietti, Phys. Rev. E 65, 055206(R) (2002). 